\section{Observations of Projectile Penetration into a TransParent \\ SoIL}

Ivan L. Guzman ${ }^{1}$, Magued Iskander ${ }^{2}$, and Stephan Bless ${ }^{3}$

Abstract: In this study, a transparent sand surrogate was employed along with high-speed imagery to un-intrusively visualize the penetration of a spherical projectile into the center of a saturated granular target, representing angular sand, at speeds ranging between 60 and $150 \mathrm{~m} / \mathrm{sec}$. The transparent sand was made by saturating an angular granular fused quartz waste product with a matched refractive index pore fluid made of sucrose. A distinct zone of opacity was observed travelling ahead of the projectile. The opacity zone appears circular during initial penetration and transitions into the shape of an elongated cone in shots with higher initial velocities. Some healing was also observed with time and some increase in transparency was observed. Some of the opacity is attributed to dilatancy of the granular fused quartz during penetration, and healing is attributed to flow of pore fluid into the dilated zone.

Key Words: Transparent soil, granular materials, penetration, dilation

\section{Introduction:}

Phenomenology associated with impact cratering onto granular and solid

\footnotetext{
${ }^{1}$ University Lecturer, New Jersey Institute of

Technology, Newark, New Jersey 07102, iguzman@njit.edu

${ }^{2}$ Professor \& Chair, Civil And Urban Engineering Department, NYU Polytechnic School of Engineering. Six Metrotech Center Brooklyn, NY 11201, Iskander@NYU.edu (Corresponding Author)

${ }^{3}$ Research Professor, NYU Polytechnic School of Engineering, sbless@NJU.edu
}

materials has been a topic of much interest in recent years $[33,44]$ and the field has also been covered in two recent review articles [36, 37]. Impact cratering has been studied both by creating numerical models to understand the penetration phenomena and experimentally. Numerical models have been used to model penetration into oblique targets [10] where the angle of impact, a more common event in nature, is different from vertical impact which is more commonly studied. Others have used existing shock physics codes to understand how the target properties affect cratering [40, 41]. Glass sphere targets in combination with gas guns and occasionally laser sheets have been also used to study crater growth during penetration into granular materials $[4,45$, 46]. Experimental techniques have also been introduced to study hypervelocity impacts into solid materials such as porous sandstone and basalt/pyrex specimens [7, 20, 28]. The penetration process through opaque materials has also been visualized by the use of a transparent observation window adjacent to the projectile trajectory, a technique commonly known as quarter space. Evolution of explosive craters in sand was extensively studied with this technique by Piekutowski [38]. The technique has also been applied to penetration into granular materials [6, 42]. However, the quarterspace technique can lead to wave reflections at the window which possibly influences the crater formation process. Although there is much data concerning penetration depth as a function of impact velocity, there are very few observations of transient craters and cavities caused by penetrating projectiles. Technically speaking a crater is a cavity that opens to the surface. 
The study of penetration events into granular media is of special interest to many engineering problems such as explosions and air blasts, earthquakes, mine blasts, vehicle and aircraft wheel loading, dynamic compaction, pile driving and rapid load testing of piles and projectile penetration. This study presents an experimental technique to non-intrusively observe the formation and development of impact cratering at all stages of penetration with the use of a transparent soil surrogate. It is important to note that impact processes in the hypervelocity regime, as those associated with cratering from a planetary perspective, require significantly higher impact velocities than the ones used in this study. However, the experimental techniques introduced herein can be modified in future studies for observation of hypervelocity impacts and the study of shock-wave induced crater formation.

Observation of crater dynamics is made possible by use of a transparent soil. Transparent soils are surrogates of natural soils that are made by matching the refractive index (RI) of particles and a saturating pore fluid to minimize refraction of light. Transparent soil surrogates have been used in model tests to study flow or deformations, and to study quasi-static penetration into sand and clay $[1,2,11,13,14,19,21-26,29-32$,
34, 39, 43]. Recently, transparent soil surrogates have also been used to assess and study depth of penetration of spherical projectiles into transparent sand [18]. In the present study, the same material was employed to visualize not only the terminal depth of penetration, but also observe the effect of the penetration on the penetrated medium. Depth of penetration, in this study, refers to penetrative low-velocity impacts such as those from meteoric impacts after velocity reduction due to the atmosphere.

In general, the term "soil" refers to natural granular earthen materials that result from the weathering of natural rock. In lieu of available natural transparent soils, this study uses a previously introduced synthetic transparent soil surrogate. The transparent soils used in this work were comprised of granular fused quartz glass, Fig. 1, combined with a refractive indexmatching liquid made of sucrose $[15,16]$. Granular fused quartz glass used herein are from the same manufacturer and have similar gradations to fused quartz used in those studies. These studies and others [12] have shown that the quasi-static rheological and geotechnical properties such as shear strength, compressibility, particle breakage and grain size distribution of these mixtures, closely resemble those of natural sands. 


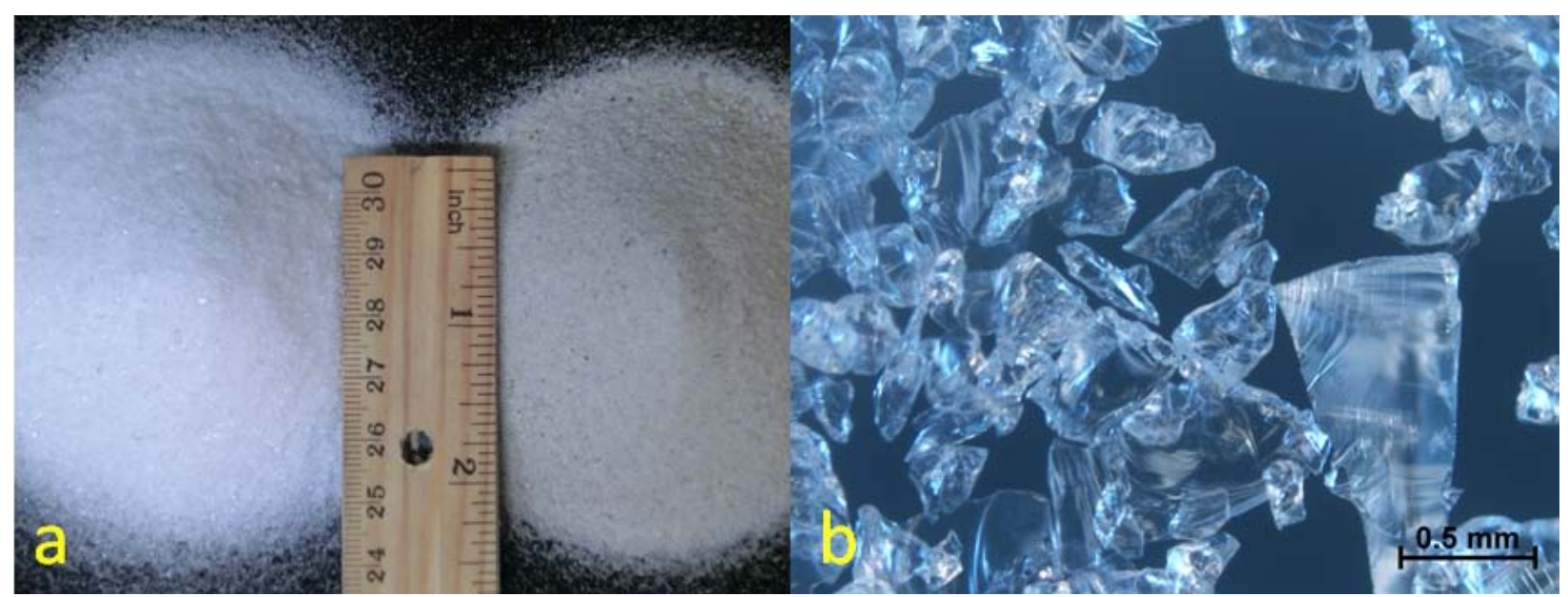

Fig. 1. Photograph of granular fused quartz (a) comparison of granular fused quartz (left) and Ottawa sand (right) (b) magnified image of granular fused quartz

\section{Experimental Setup}

\subsection{Materials}

Granular fused quartz used in this study had a gradation of particles that passed the \#20 sieve and were retained on the \#60 sieve [3] and is thus designated as FQ(-20+60), or simply FQ. The maximum and minimum dry densities of FQ were determined to be 1.30 and $1.00 \mathrm{~g} / \mathrm{cm}^{3}$, respectively. The axial stress versus axial strain, and axial stress versus volumetric strain of granular fused quartz under triaxial compression is shown in Fig. 2. It can be seen that sucrose-saturated granular fused quartz under low confining pressure exhibits a highly dilative response during axial compression. Dilatancy in this study refers to an increase in total volume due to a change in shape. The particle size distribution for FQ and other natural soils is presented in Fig. 3.

The state of compaction of granular materials is commonly characterized by using the relative density scale. This refers to the relative compaction of a granular material at the time of testing in relation to its maximum and minimum compaction achievable with standardized laboratory procedures. Relative density has a scale of 100 (most compact) to $0 \%$ (most loose), with generally agreed subdivisions of $100-80 \%$ (very dense), 80-60\% (dense), 60-40\% (medium dense), 40-20\% (loose), and 20-0\% (very loose). 

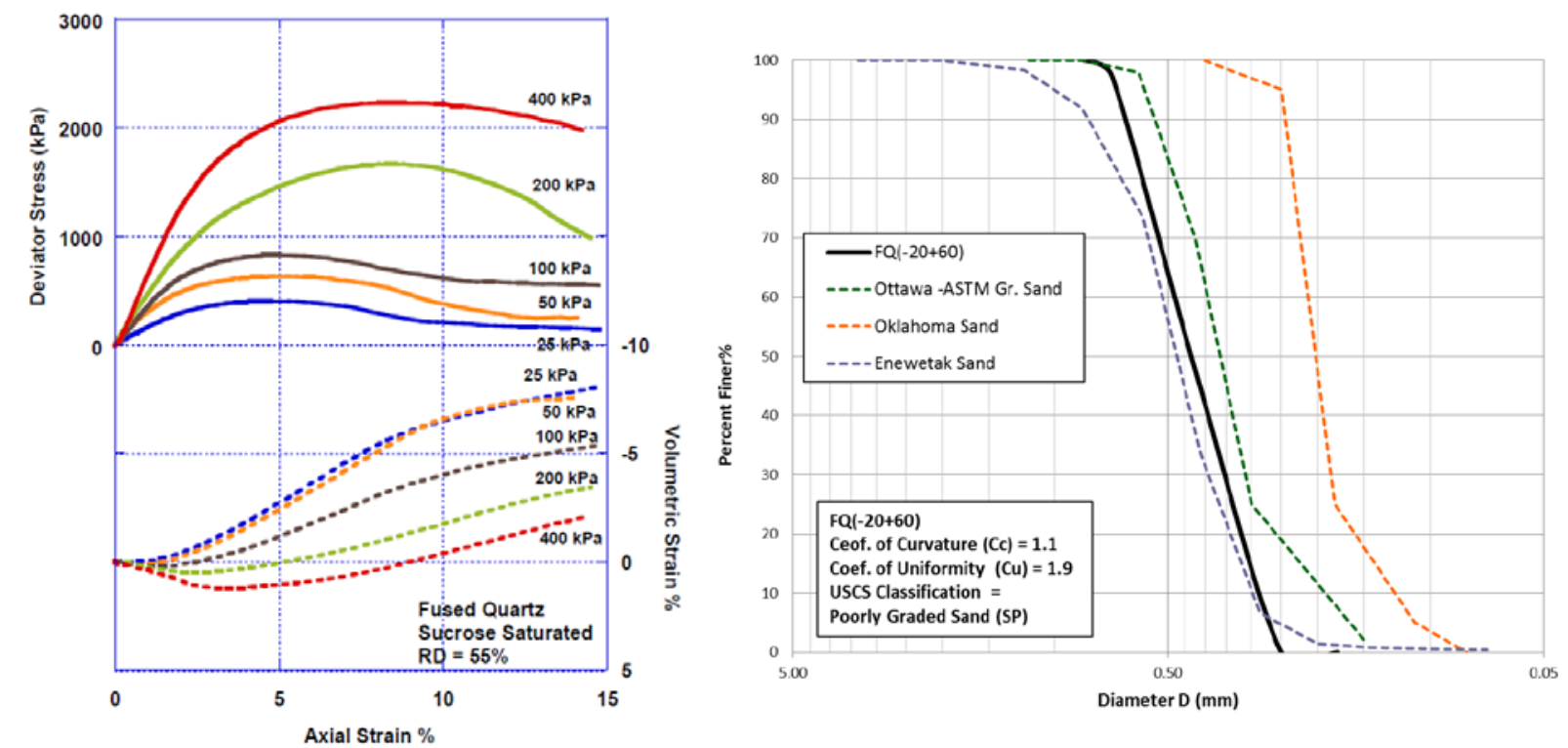

Fig. 2. (left) Stress versus axial strain, and volumetric strain versus axial strain curves of sucrose saturated granular fused quartz under triaxial compression, contractive volume change is positive, dilative is negative.

Fig. 3. (right) Gradation curve for granular fused quartz, $F Q(-20+60)$ and other commercially available natural sands.

Transparent targets were prepared by pluviating (raining) FQ into a container half full with the saturating fluid. The technique of pluviating through several inches of saturating fluid serves two purposes; first, it creates a soil structure that mimics natural sedimentation at a controlled relative density; and second, the presence of a standing layer of liquid "filters" the air bubbles, a major source of transparency degradation, which improves the transparency of the specimens. Pluviation through the pore fluid produced very loose specimens with a relative density on the order of 10 to $15 \%$. Dense specimens were prepared by using an electro-mechanical vibrator (Fritsch model Analysette 3 Pro), operated at an amplitude of $0.1-0.4 \mathrm{~mm}$ until the desired relative density was achieved. To ensure correct densities, vibration was conducted until a known quantity of soil occupied a predetermined volume previously marked on the transparent containers. Weight measurements of the container plus saturating fluid were recorded prior to and after pluviation to determine the volume fraction of solids deposited and thus the dry density, and relative density of the model soil. The total (bulk) density was then calculated by assuming $100 \%$ saturation. Dry density is defined as the weight of the solid portion of the soil structure (granular particles) divided by the total volume of the soil structure, which is constant regardless of saturation conditions (dry or wet).

\subsection{Projectile Accelerator and Projectiles}

Projectiles used in this study consisted of Type 316 stainless steel $(18 \%$ chromium and $8 \%$ nickel) spheres with a diameter of $9.9 \mathrm{~mm}$ (0.39 in), with a density of $7,600 \mathrm{~kg} / \mathrm{m}^{3}$ which yields mass to diameter ratios of $0.39 \mathrm{~kg} / \mathrm{m}$. 
An electro-pneumatic projectile accelerator (air gun), [8], was used to propel projectiles at approximate entry velocities of 60,100 and $150 \mathrm{~m} / \mathrm{s}$. Projectile exit velocities were measured using a custom made optical speedometer, attached to the end of the barrel [8]. The speedometer consisted of three infrared photo-sensor pairs fixed 30 $\mathrm{mm}$ apart mounted on a $13 \mathrm{~mm}$ diameter and $150 \mathrm{~mm}$ long extension attached to the end of the gun barrel. Thus, the distance between sensor 1 \& 2, and, sensor $2 \& 3$ was $30 \mathrm{~mm}$ and the distance between sensor $1 \& 3$ was $60 \mathrm{~mm}$. The average speed of a projectile passing through the tube was measured by dividing the distance between two pairs of photo-sensors by the amount of time in between each sensor's status change. This resulted in three reported speeds, which provided a measure of redundancy. The probable uncertainty of the velocity measurements was found by employing Taylor's theorem. For speeds of 60, 100, and $150 \mathrm{~m} / \mathrm{s}$ the probable uncertainty is approximately $1.1 \%, 1.9 \%$, and $2.8 \%$, respectively. The velocity was also confirmed by measuring the distance traveled by the projectile before impact in between camera frames, divided by the time between frames. The percent difference between the velocities measured by the speedometer and the ones measured between frames were $3.6 \%, 0.0 \%, 1.0 \%$, respectively. These measurements also confirm that the dropoff in velocity was not significant between the end of the barrel and target.

Several precautions were implemented to reduce the influence of the "air blast" on the specimen prior to projectile penetration. First, the distance from the end of the barrel to the surface of the soil target was $>23 \mathrm{~cm}$ in all tests, which allows for the air travelling ahead of, or behind, the projectiles to disperse after exiting the barrel and before reaching the soil target. Second, a plastic lid with a $25 \mathrm{~mm}$ orifice was employed to cover the top of the soil target container, which reduces the amount of "air blast" that can reach the target surface.

The depth of penetration (DOP) was measured by observing the distance between the original top of the soil and the final resting position of the projectile. A linear laser displacement gage (LLDG), was used to record the depth of penetration measurements.

Table 1 Summary of test conditions and results of projectiles shot into the center (half space) of transparent soil targets

\begin{tabular}{lllllll}
\hline \hline Test & Granular Material & $\begin{array}{l}\text { Relative } \\
\text { Density } \\
\%\end{array}$ & $\begin{array}{l}\text { Dry } \\
\text { Density } \\
\left(\mathbf{g} / \mathbf{c m}^{\mathbf{3}}\right)\end{array}$ & $\begin{array}{l}\text { Total } \\
(\mathbf{b u l k}) \\
\text { Density } \\
\left(\mathbf{g} / \mathbf{c m}^{3}\right)\end{array}$ & $\begin{array}{l}\text { Initial } \\
\text { Velocity } \\
(\mathbf{m} / \mathbf{s})\end{array}$ & $\begin{array}{l}\text { Terminal } \\
\text { Depth of } \\
\text { Penetration } \\
(\mathbf{c m})\end{array}$ \\
\hline \hline M-1 & FQ(-20+60) - Sucrose & 65 & 1.18 & 1.80 & 66.3 & 3.2 \\
$M-2$ & $F Q(-20+60)-$ Sucrose & 69 & 1.19 & 1.80 & 104.6 & 4.8 \\
$M-3$ & $F Q(-20+60)-$ Sucrose & 65 & 1.18 & 1.80 & 149.5 & 8.7
\end{tabular}




\section{$2.3 \quad$ Optical Setup}

Images were captured using a NAC Image Technology, Memrecam HX-5 high-speed digital camera at a frame rate of 10,000 frames per second. The targets were back lit with the use of one 250 and one 500 watt tungsten lamps. The lamps were oriented to illuminate a white reflective wall which provided silhouette images of the sample. This technique resulted in adequately uniform background brightness. This indirect lighting technique is also desirable because of the heat radiation that is natural to tungsten lights, which when oriented directly into the target can increase the temperature of the transparent soil samples, thus affecting transparency of the target which depends on perfect matching of the temperature-sensitive RI of the saturating fluid and that of fused quartz.

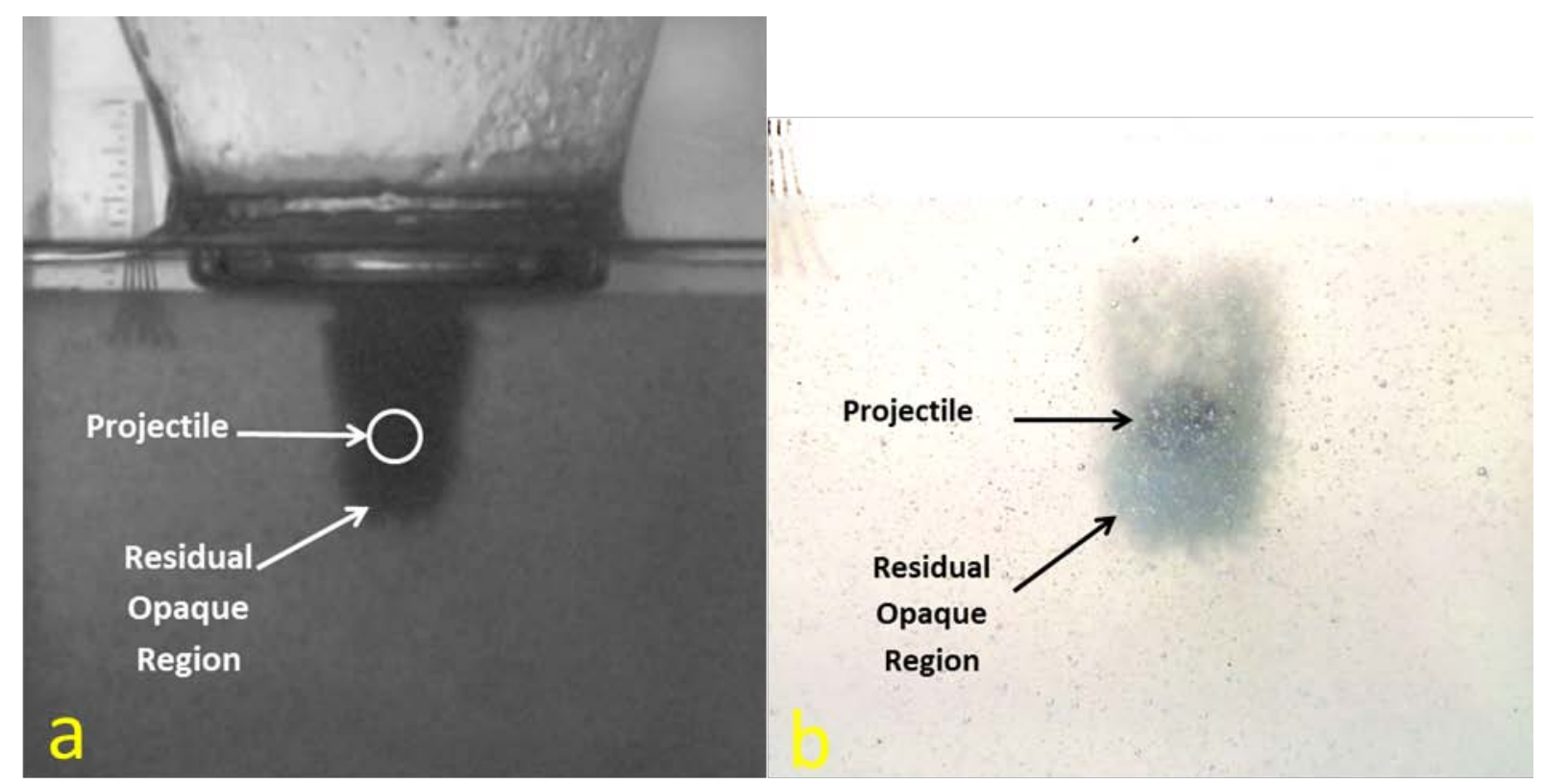

Fig. 4. Images of test in the center of the sample into transparent sucrose saturated granular fused quartz $(60 \mathrm{~m} / \mathrm{s})$. a) Snapshot from high speed camera near the end of the penetration. b) Picture of penetration event after the cavity has had a chance to heal. Although the projectile is not visible by the high-speed camera during penetration, after a certain degree of cavity healing it can be distinguished as shown in the picture 
This opaque region was also observed in shots taken against an observation window (quarter space) into sucrose saturated FQ, Fig. 5a. In those experiments the target was front-lit, and the area where transparency has been lost appears white. In all tests the opaque region shrunk somewhat, i.e. regained transparency, after the projectile stopped, as shown in Fig. 4b and $5 \mathrm{~b}$. After shrinking the opaque region will be referred to as residual opaque region.

\subsection{Shape of the Opaque Region}

The shape and size of the opaque region and residual opaque region observed with the high speed camera is shown in selected sequential images in Fig. 6. It was found that the opaque regions in sucrose saturated FQ in tests with initial speeds of 60 and $100 \mathrm{~m} / \mathrm{s}$ have a hemispherical shape soon after impact, which elongates as penetration proceeds. The initial hemispherical shape is very likely due to the spherically-divergent shock wave generated at impact; later the projectile emerges from the bottom of the shock-induced initial opaque region, after which the opaque region is symmetric around the penetration channel. We believe that the shock wave induced stress transient has caused the material to loose transparency (opaque region), probably due to cavitation by the relative motion of particles and fluid, or the dilatancy due to the hoop strain associated with a diverging stress wave. We note that there are several observations of impact induced compaction waves in granular materials at these types of velocities $(5,6)$. In the test with initial velocity of approximately $150 \mathrm{~m} / \mathrm{s}$ the elongated hemispherical shaped opaque region begins to take the shape of a cone at the end of penetration, which is more pronounced after the region heals. During deep penetration, the opaque region probably represents contours of a specific strain level. Several recent studies of in situ deformation in transparent soils show these kinds of contours, $[9,27,35]$. In all of the tests, after the opaque region reaches its maximum depth it then recedes somewhat, Fig. 6 . Near the surface, there is a reduction in the residual size of the crater as some sucrose saturated granular fused quartz fills part of the transient crater due to gravity, and is visible posttest due to the presence of air bubbles trapped in the ejecta. The visible transparent layer above the targets is the excess pore fluid above the transparent soil surrogate. It is small enough that it's displaced by air travelling ahead of the projectile before impact, thus not affecting to great extent the impact velocity of the projectile. 

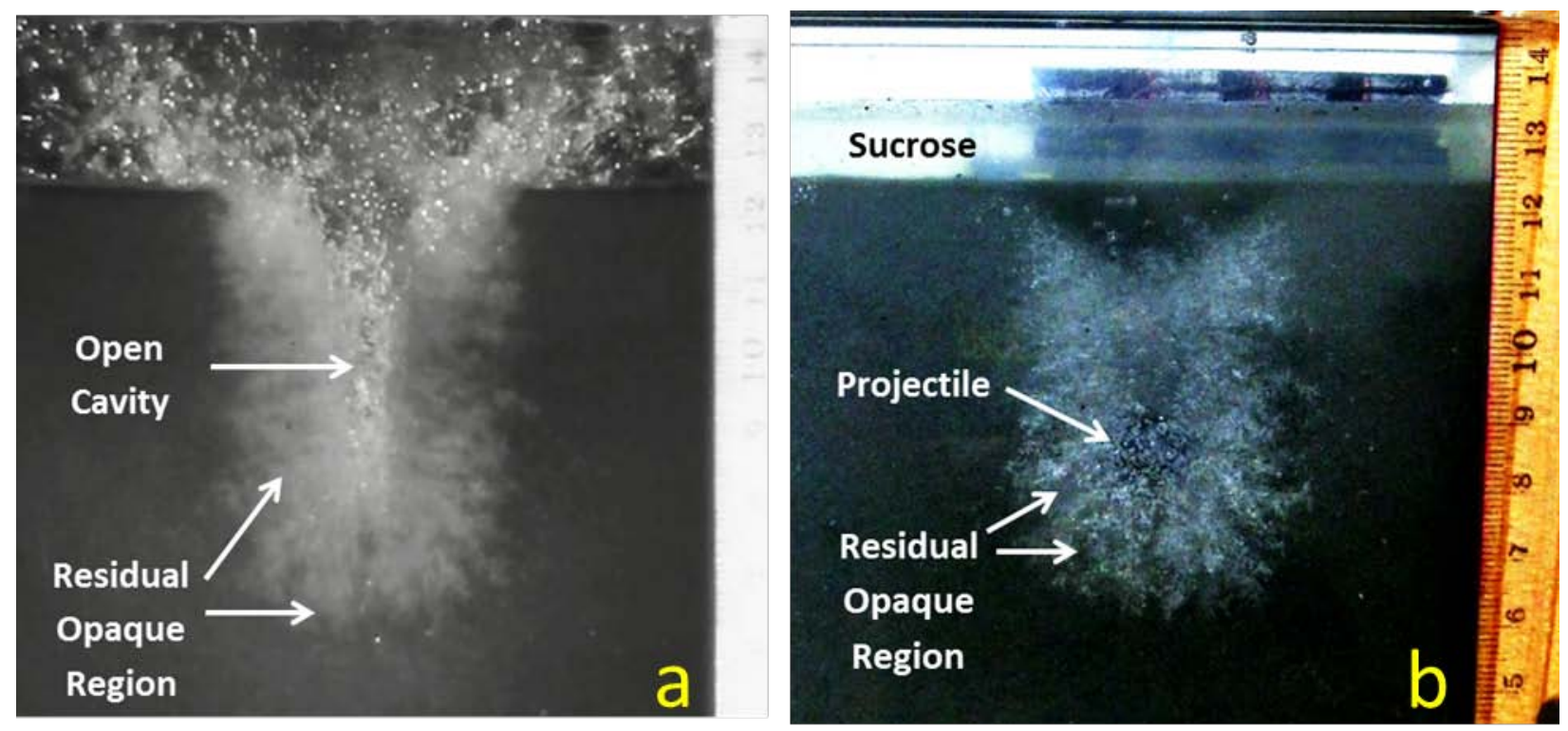

Fig. 5. Images of test against an acrylic observation window on sucrose saturated granular fused quartz $(100 \mathrm{~m} / \mathrm{s})$. a) Snapshot from high speed camera near the end of penetration. b) Picture of the same penetration event after the cavity has had a chance to heal following the penetration event

During penetration tests in the center of targets the opaque region ahead of the projectile can be seen through the elsewhere-transparent soil. A frame by frame analysis of each of the tests listed in Table 1 yields data to construct a plot of depth vs. time, Fig. 7. The advancement of the opaque region increases with time, reaches a maximum, and then recedes leaving a residual opaque region. Terminal depths of penetration of the projectile, for all tests, are plotted and represented by large colored circles (drawn to scale) in Fig. 7. It can be seen that the residual opaque region extends deeper than the projectile by about 1.5 to 2.0 diameters.

The data in Fig. 7 was differentiated with respect to time to obtain the instantaneous velocity of the movement of the bottom of the opaque region, Fig. 8. It can be seen that the edge of the opaque region at the beginning of the penetration event accelerates to a speed higher than the speed of the projectile. This is the reason why we cannot see the tip of the projectile, because the opaque region is moving faster than the projectile. The initial acceleration is followed by a deceleration episode that is then followed by smaller peaks which represent smaller cycles of acceleration and deceleration. Surprisingly, the smallest amount of opaque region retrenchment takes place in the highest velocity shot.

\subsection{Origin of the Opaque Region}

Expansion of a spherical or cylindrical cavity gives rise to a region in which hoop strain becomes tensile; this may cause dilation. The difference between the compressive axial strain and tensile hoop strain also generates large shear strains. Granular fused quartz is a highly dilative material due to its angular nature [15, $16]$, and when tightly packed and subjected to low confining pressure, and sheared will exhibit a highly dilative response which will cause particle arrangements to expand. The dilative 
tendency of granular fused quartz is evidenced through conventional quasi static triaxial stress strain tests conducted on granular fused quartz which depict marked dilation at confining pressures ranging from $50-400 \mathrm{kPa}$, Fig 2.

In order for the targets to make room for the fast moving projectile, particles will have to come out of their tightly packed structure, which leads to dilation. During penetration the area of dilation is visible because when the soil structure dilates at a high speed there is not enough time for the pore fluid to move into and fill the newly created voids. In the absence of pore fluid, the dilation region may then be partially filled with air or vapor (due to cavitation). For the former, air may be introduced from the cavity behind the projectile. For the latter case, where the void is not filled with air, it is speculated that cavitation takes place. During cavitation the voids between particles are partially filled with vapor. Both air and vapor have a different RI than that of the pore fluid, resulting in opacity within that region. Opacity due to the change in the RI prevents the light coming from the opposite side of the target to reach the camera lens.

At the end or near the end of penetration, the newly created void space will either collapse after the event or is eventually filled with the surrounding pore fluid or air. This phenomena can be observed in all of the sucrose saturated FQ tests shot in the center of the sample (half space). In these tests the maximum opaque region is seen near the end of the penetration event, at which time the region is partially filled with vapor, air or a combination of both, but is then followed by a partial contraction of the visible opaque region, Fig. 9.a. It is believed that contraction of the opaque region is caused primarily by flow of pore fluid into a zone that was shear or hoop strain dilated during the penetration event. Air trapped in the residual opaque region becomes a permanent marker of a section of the open cavity and part of the dilation area that does not fill up with pore fluid after the event.

When targets are observed after the shot, the residual opaque regions appear surrounded by a spongy material that is clearly due to air mixed into the pore fluid-particle suspension. However, the portion of the opaque region that recedes almost immediately after the projectile stops is plausibly due to shear or hoop strain-induced dilation, and recovery of transparency is explained by seepage of pore fluid into the voids created by the dilative expansion associated with cavity formation.

Pressure or temperature sensitivity of the index of refraction of sucrose could also cause loss of transparency. However, one would not expect pressure or temperature-induced index changes to persist after passage of the projectile, and they should become small as the projectile comes to rest, which was not observed. Thus, we do not believe that the opaque region is due to pressure or temperature-dependent changes in the index of refraction of the materials involved. 


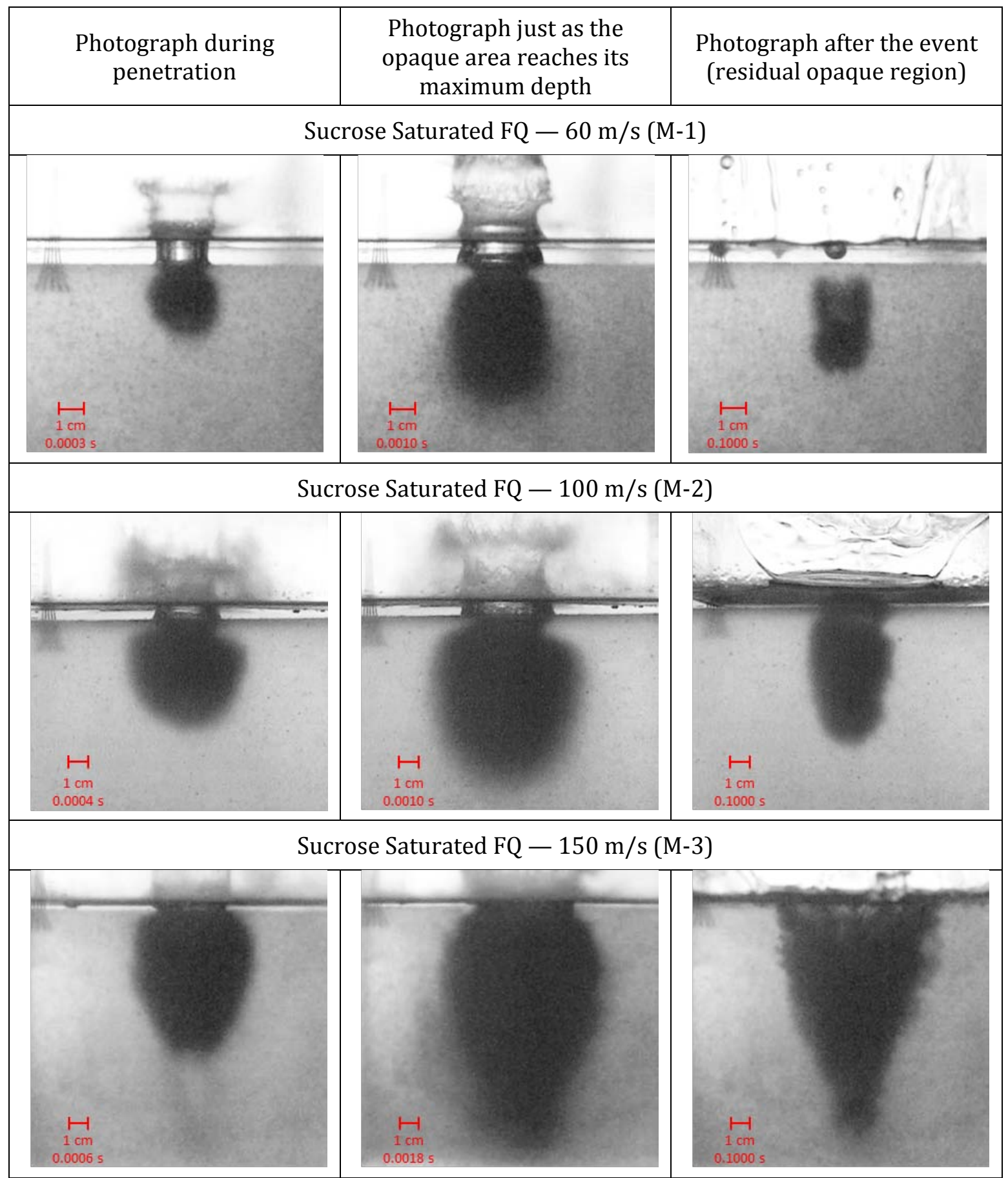

Fig. 6. Progressive snapshots of penetration into transparent sucrose saturated granular fused quartz shot at the center of the target, from [17] 


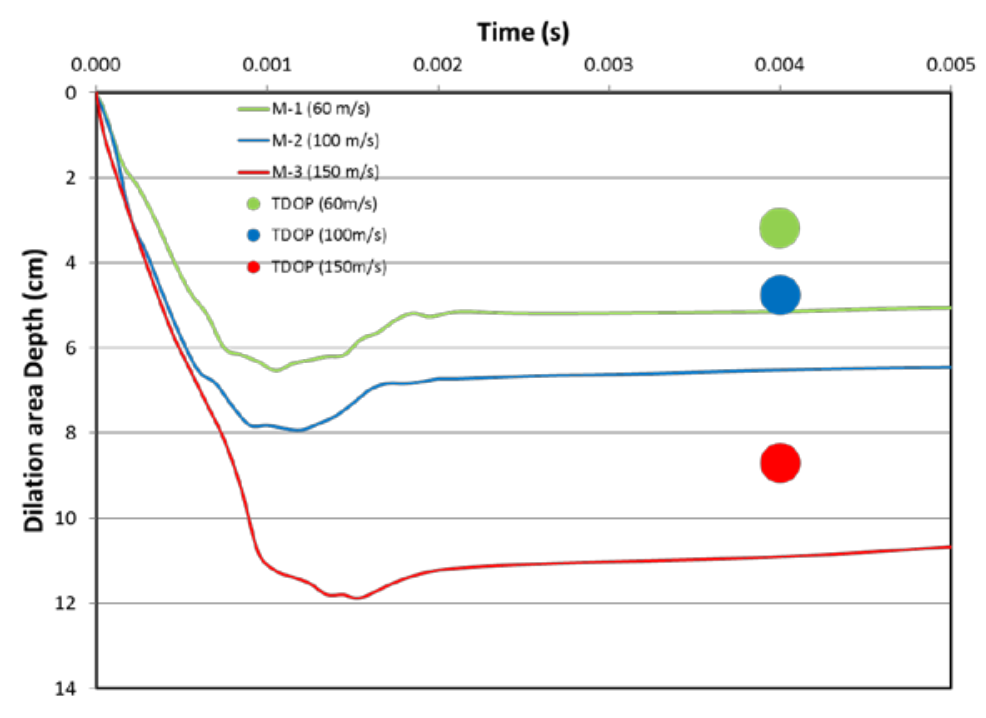

Fig. 7. Depth of opaque region vs. time, for projectiles shot at the center of the target into sucrose saturated granular fused quartz. Terminal DOPs (TDOP) for each test are presented, to scale, with color circles

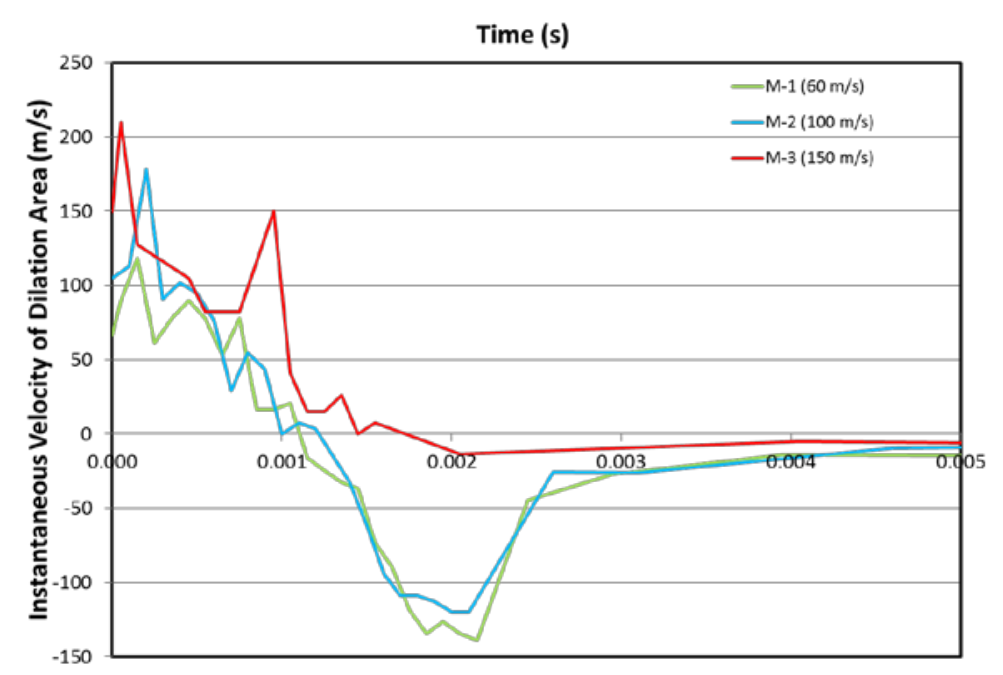

Fig. 8. Instantaneous velocity of the depth of opaque region vs. time, for projectiles shot in the center of the target into sucrose saturated granular fused quartz 

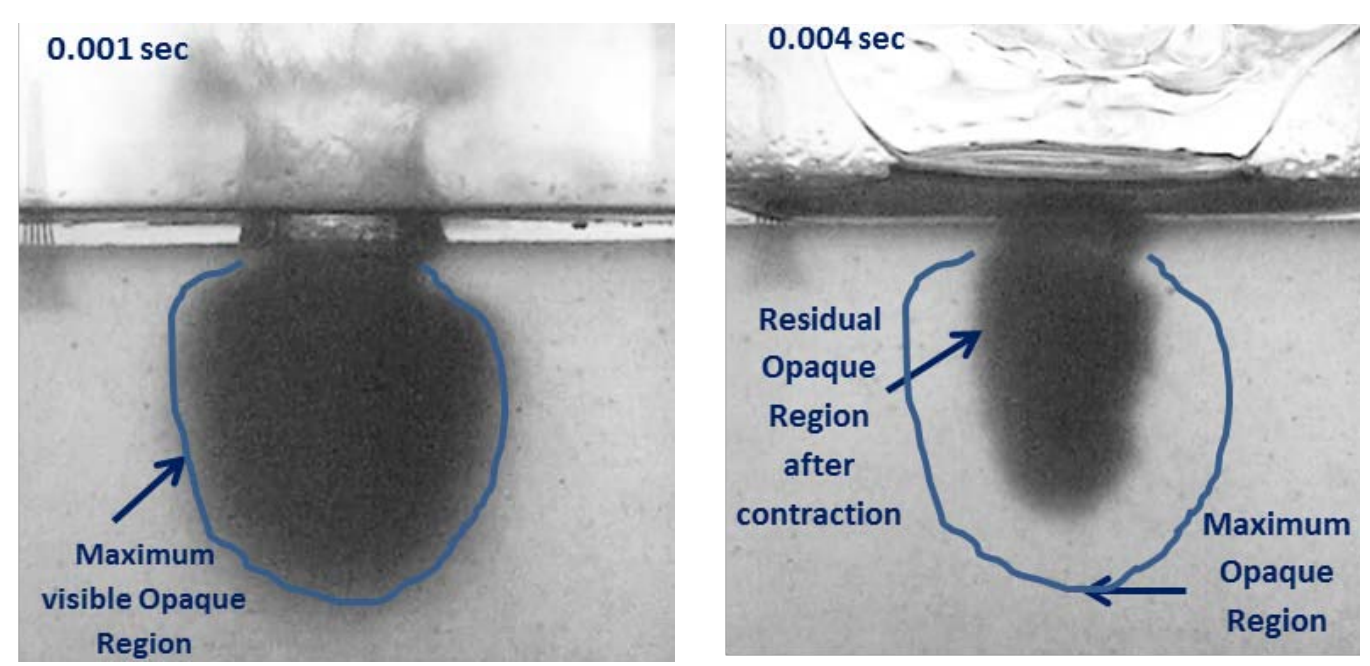

Fig. 9. Opaque region for test M-2. (a) Maximum visible opaque region. (b) Residual opaque region after contraction of dilated zone due to collapse, or saturation with pore fluid

\section{$4 \quad$ Discussion}

During projectile penetration through transparent soil targets a visible opaque region was observed ahead and around the projectiles. The opaque zone appears circular during initial penetration and transitions into the shape of an elongated oval, which can become almost a cone in shots with higher initial velocities. A certain degree of healing happens immediately after penetration, where the opaque region is reduced. The opaque region reaches its maximum at some point during the end of the penetration event, followed by a contraction of the visible opaque region in which the region that was not filled with air has a chance to collapse or fill with pore fluid. The behavior of the apparent crater is consistent with shear or hoop strain induced dilation followed by flow of pore fluid to saturate the porous medium. After the opaque region has had time to heal there is a permanent residual opaque region that extends about 1.5 to 2.0 diameters ahead of the projectile, where air that entered the target with the projectile fills the pores and inhibits transparency.
This behavior is consistent with the behavior of densely packed angular soils when subjected to shear stresses. However, the shape and size of the observed opaque region might be different when the target soil is comprised of loose rounded particles. For this case, we expect a much smaller dilation area replaced by compaction of the granular material ahead and around the projectile, which would likely not be visible using this technique. The observations presented herein highlight the importance of the relative density and the angular nature of the soil when studying penetration events into granular materials. In lieu of a dilation area traveling ahead of the projectile the projectile near field would be visible during the penetration event.

The techniques presented herein are in their early stages of development and are not yet applicable to hypervelocity studies, mainly due to physical constraints of many laboratories. However, the use of transparent soil surrogates made of fused quartz and sample preparation techniques used 
herein can be used in hypervelocity studies to visualize penetration into a granular medium when combined with an adequate hypervelocity gun and a much faster camera equipped with high end optics. This technique when properly applied to natural hypervelocity impacts can shed light on components of the penetration event. Depending on the size and shape of the projectile, the characteristics of the target and impact speed, the projectile could be visible during penetration, thus, projectile disruption, projectile fragmentation, and near field behavior can be studied as well as others like cavity expansion due to shock acceleration of the target.

\section{Conclusions}

The use of transparent soil surrogates and a high-speed camera allows us to observe and study a projectile penetration event into the middle of a transparent soil target un-intrusively. There is a distinct dilation-related opaque zone that travels ahead of a spherical projectile, which inhibits transparency and does not allow a frame by frame analysis of the exact location of the projectile during penetration. Thus, it was possible to investigate the far field area ahead of the projectile during penetration but not the near field area near the tip of the projectile, where the projectile cannot be seen.

The technique permits observing target response that is not visible with opaque targets. It is believed that similar mechanisms take place during penetration of natural sands, but that they are difficult to discern due to the target opacity.

\section{Acknowledgements}

The authors gratefully acknowledge the support of the Defense Threat Reduction Agency Grant No: HDTRA1-10-1-0049 and The United States National Science Foundation Grant No: DGE 0741714. Fused quartz powder used in this investigation was manufactured by Mineral Technology Corporation (Mintec). Low Color Sucrose $\mathrm{TM}^{\mathrm{TM}}$ used to match the granular fused quartz was manufactured by Indiana Sugars. The HX5 high-speed camera was manufactured by NAC Image Technology Inc.

\section{References}

1. Ahmed $M$, Iskander $M$ (2011) Analysis of tunneling-induced ground movements ssing transparent soil models. Journal of Geotechnical and Geoenvironmental Engineering, ASCE 137:525-231

2. Ahmed $M$, Iskander $M$ (2011) Evaluation of tunnel face stability by transparent soil models. Tunneling and Underground Space Technology 27:101-110

3. ASTM-C136-06 (2006) Standard test method for sieve analysis of fine and coarse aggregates.

4. Barnouin-Jhaa OS, et. Al (2007) Non-intrusive measurements of crater growth. Icarus 188:506-521

5. Borg JP, Morrissey M, Perich C, Vogler T, Chhabildas L (2013) In situ velocity and stress characterization of a projectile penetrating a sand target: Experimental measurements and continuum simulations, International Journal of Impact Engineering 51:23-35

6 Borg JP, Vogler JT (2008) An experimental investigation of a high 
velocity projectile penetrating sand. XIth International Congress and Exposition, Society of Experimental Mechanics, Inc., Orlando, Florida, USA

7. Buhl E (2012) Deformation of dry and wet sandstone targets during hypervelocity impact experiments, as revealed from the MEMIN Program. Meteoritics \& Planetary Science 48:71-86

8. Cave A, Roslyakov S, Iskander M, Bless S (2014) Design and performance of a laboratory pneumatic gun for soil ballistic applications. Experimental Techniques, Society of Experimental Techniques:1-13. doi: 10.1111/ext.12091

9. Chen $Z$, Omidvar $M$, Iskander $M$, Bless $S$ (2014) Modelling of projectile penetration using transparent soils. International Journal of Physical Modeling in Geotechnics 14:3:68-79

10. Elbeshausen D, Wunnemann K, Collins GS (2009) Scaling of oblique impacts in frictional targets: implications for crater size and formation mechanisms. Icarus 204:716-731

11. Ezzein FM, Bathurst RJ (2011) Development of a geosynthetic pullout test apparatus with transparent granular soil. Pan-Am CGS Geotechnical Conference, Ontario, Canada

12. Ezzein FM, Bathurst RJ (2011) A transparent sand for geotechnical laboratory modeling. ASTM Geotechnical Testing Journal 34:1-12
13. Fernandez Serrano $R$, Iskander $M$, Tabe K (2011) 3D Contaminant flow imaging in transparent granular porous media. Geotechnique Letters $1: 1-8$

14. Gill D, Lehane B (2001) An optical technique for investigating soil displacement patterns. ASTM Geotechnical Testing Journal 24:324329

15. Guzman IL, Iskander M (2013) Geotechnical properties of sucrosesaturated fused quartz for use in physical modeling. Geotechnical Testing Journal 36:8. doi: 10.1520/GTJ20120182

16. Guzman IL, Iskander $M$, SuescunFlorez E, Omidvar M (2013) A transparent aqueous-saturated sand surrogate for use in physical modeling. Acta Geotechnica. doi: 10.1007/s11440-013-0247-2

17. Guzman IL, Iskander M (2014) Observations of projectile penetration into transparent soils. http://www.youtube.com/watch?v= L-8GazoYNgY

18. Guzman IL, Iskander M, Bless S, Qi C (2014) Terminal depth of penetration of spherical projectiles in transparent granular media. Granular Matter. doi: 10.1007/s10035-014-0528-y

19. Hird CC, Stainer SA (2010) Modelling helical screw piles in clay using a transparent soil. In: Proceedings of the 7th Annual International Conference on Physical Modeling in Geotechnics, Zurich, Switzerland. International Society for Soil Mechanics and Geotechnical Engineering, p 6 
20. Hoerth T, et al (2013) Hypervelocity impacts on dry and wet sandstone: Observations of ejecta dynamics and crater growth. Meteoritics \& Planetary Science 48:23-32

21. Iskander M, Lai J, Oswald C, Mannheimer R (1994) Development of a transparent material to model the geotechnical properties of soil. ASTM Geotechnical Testing Journal 17:425-433

22. Iskander M (1998) Transparent soils to image 3D flow \& deformation. In: 2nd International Conference on Imaging Technologies: Techniques and Applications in Civil Engineering: ASCE, pp 255-264

23. Iskander M, Sadek S, Liu J (2003) Optical measurement of deformation using transparent silica gel to model sand. International Journal of Physical Modeling in Geotechnics 2:13-26

24. Iskander M (2010) Modelling with transparent soils, visualizing soil structure interaction and multi phase flow, non-intrusively. Springer

25. Iskander M, Liu J (2010) Spatial deformation measurement using transparent soil. Geotechnical Testing Journal 33:1-7

26. Iskander M, Liu JY, Sadek S (2002) Transparent amorphous silica to model clay. Journal of Geotechnical and Geoenvironmental Engineering 128:262-273

27. Iskander $\mathrm{M}$, Bless $\mathrm{S}$, Omidvar $\mathrm{M}$ (2015) Rapid Penetration into Transparent Media. Elsevier

28. Kimberly J, Ramesh KT (2013) Visualization of early stage damage propagation during hypervelocity impacts on brittle materials. Proceedings of the 12th Hypervelocity Impact Symposium . Procedia Engineering 58:678-683

29. Lehane B, Gil D (2004) Displacement fields induced by penetrometer installation in an artificial soil. International Journal in Geotechnics 4:25-36

30. Liu J, Iskander MG (2010) Modelling capacity of transparent soil. Canadian Geotechnical Journal 47:451-460

31. Liu JY, Iskander M (2004) Adaptive cross correlation for imaging displacements in soils. ASCE Journal of Computers in Civil Engineering 18:12

32. Lo HC, Tabe K, Iskander M, Yoon SH (2010) A transparent water-based polymer for simulating multiphase flow. ASTM Geotechnical Testing Journal 33:1-13

33. Melosh, HJ (1989) Impact cratering: A geologic process. Research supported by NASA. New York, Oxford University Press (Oxford Monographs on Geology and Geophysics, No. 11), 1989, 253 p.

34. Ni Q, Hird C, Guymer I (2010) Physical modelling of pile penetration in clay using transparent soil and particle image velocimetry. Geotechnique 60:121132

35. Omidvar $M$, Doreaa Malioche J, Chen Z, Iskander M, Bless S (2015) Visualizing kinemativs of dynamic penetraion in granular media using transparent soils. ASTM Geotechnical Testing Journal 38:5:117 
36. Omidvar $\mathrm{M}$, Iskander $\mathrm{M}$, Bless $\mathrm{S}$ (2012) Stress-strain behavior of sand at high strain rates. International Journal of Impact Engineering 49:192-213

37. Omidvar $\mathrm{M}$, Iskander $\mathrm{M}$, Bless $\mathrm{S}$ (2014) Response of granular media to rapid penetration. International Journal of Impact Engineering 66:6082.doi:10.1016/j.ijimpeng.2013.12.0 04

38. Piekutowski AJ (1980) Formation of bowl-shaped craters. Eleventh Lunar Planetary Science Conference, Houston, Texas pp. 2129-2144

39. Sadek S, Iskander MG, Liu J (2002) Geotechnical properties of transparent silica. Canadian Geothecnical Journal 39:111-124

40. Senft LE (2009) The effect of target properties on impact cratering. Harvard University, Doctoral dissertation, 197 pages

41. Senft LE, Stewart ST (2009) Dynamic fault weakening and the formation of large impact craters Earth Planet Science Letters 287:471-482

42. Shultz PH, et. al. (2005) Expectations for crater size and photometric evolution from the deep impact collision. Space Science Reviews 117:207-239

43. Tabe K, Iskander M, Honma S (2011) Transparent aquabeads to visualize flow in porous material. Advanced Materials Research 239-242:26022605

44. Turtle EP, Pierazzo E, Collins GS, Osinski GR, Melosh HJ (2005) Impact structures: what does crater diameter mean? . Geological Society of America, Special Papers 384, large Meteorite Impacts III:1-24

45. Yamamoto S (2006) Transient crater growth in granular targets: An experimental study of low velocity impacts into glass sphere targets. Icarus 183:215-224.

46. Yamamoto $S$, Bornouin-Jha OS, T. T, Sugita S, Matsui T (2009) An empirical model for transient crater growth based on direct observations. Icarus 203:310-319 